\title{
"DIGA-ME QUANTOS TE CITAM, E EU TE DIREI QUEM ÉS": ESTUDO SOBRE AS CITAÇÕES NO ÂMBITO DA PESQUISA CONTÁBIL BRASILEIRA
}

\author{
Sandro Vieira Soares 1 \\ Silvia Pereira de Castro Casa Nova ${ }^{2}$ \\ Raimundo Nonato Lima Filho ${ }^{3}$
}

- Artigo recebido em: 04/02/2020 -- Artigo aceito em: 20/05/2020 -- Segunda versão aceita em: 12/09/2020

\section{RESUMO}

Uma perspectiva teórica afirma que os diversos motivos para que artigos sejam citados transitam entre dois polos denominados universalista e socialconstrutivista. Características, como originalidade dos resultados e rigor metodológico, são classificadas como fatores do polo universalista; enquanto características, como fama do autor e prestígio do periódico, são classificadas como do polo social-construtivista. Diante dessa perspectiva teórica, coloca-se a questão de pesquisa: quais fatores do polo social-construtivista influenciam as citações que um artigo sobre Contabilidade recebe no âmbito da pesquisa contábil brasileira? Para responder essa questão, fez-se uma coleta de dados de 2.540 artigos, publicados por revistas brasileiras de contabilidade, entre os anos de 2007 e 2012, realizando-se, para a análise desses dados, uma regressão binomial negativa. Com base na revisão de literatura, foram elaboradas 16 hipóteses de fatores que influenciam a quantidade de citações dos artigos. As hipóteses sobre a influência dos fatores afiliação institucional do primeiro autor, revista em que foi publicado, linha de pesquisa e número de referências foram aceitas. Conclui-se, portanto, que, no âmbito da pesquisa contábil brasileira, as citações recebidas por um artigo sofrem influência da afiliação institucional do

\footnotetext{
1 Doutor em Controladoria e Contabilidade pela Universidade de São Paulo (USP). Possui PósDoutorado em Administração pela Universidade do Sul de Santa Catarina (UNISUL). Professor permanente da Universidade do Sul de Santa Catarina (UNISUL). Endereço: Rua Antônio Dib Mussi, 366, Centro, Florianópolis, SC, Brasil, CEP: 88015-110. E-maill: sandrovs@usp.br https://orcid.org/0000-0001-7076-4936

2 Professora titular da Universidade de São Paulo (USP). Pesquisadora visitante na Université Laval, no ISCTE/IUL e na University of Minnesota. Pós-doutora pela Fundação Getúlio Vargas. Doutora e mestre em Controladoria e Contabilidade pela Universidade de São Paulo (USP). Endereço: Av. Prof. Luciano Gualberto, 908, Butantã, São Paulo/SP, Brasil, CEP: 05508-010, Email: silvianova@usp.br

https://orcid.org/0000-0003-1897-4359

3 Doutor em Controladoria e Contabilidade pela Universidade de São Paulo (USP) e Doutor em Administração pela Universidade Federal da Bahia (UFBA). Possui Pós-Doutorado pela Universidade Federal da Paraíba (UFPB). Professor da Universidade de Pernambuco (UPE) e da Autarquia Educacional do Vale do São Francisco (AEVSF). Endereço: Campus Universitário s/n, Vila Eduardo, Petrolina/PE, Brasil, CEP: 56328-903. E-mail: rnlfilho@gmail.com https://orcid.org/0000-0003-4953-5260
}

Editor responsável pela aprovação do artigo: Dr. Eduardo Mendes Nascimento Editora responsável pela edição do artigo: Dr ${ }^{a}$. Bruna Camargos Avelino 
"Diga-me Quantos te Citam, e Eu te Direi Quem és": Estudo Sobre as Citações no Âmbito da Pesquisa Contábil Brasileira

primeiro autor, da revista em que o artigo foi publicado, da linha de pesquisa a que pertence e do número de referências, ou seja, fatores relacionados ao polo social-construtivista. A aceitação apenas parcial e a rejeição das demais hipóteses revelam que o cenário da pesquisa contábil brasileira se mostra flexível quando comparado ao cenário de pesquisa internacional.

Palavras-chave: Citação, Periódicos científicos, Pesquisa científica, Contabilidade, Pesquisa contábil, Fator de impacto.

\title{
"TELL ME HOW MANY QUOTE YOU, AND I'LL TELL WHO YOU ARE": STUDY ABOUT CITATIONS IN THE CONTEXT OF BRAZILIAN ACCOUNTING RESEARCH
}

\begin{abstract}
There is a theoretical perspective that maintains that the various reasons that lead to articles being cited fall between two poles called universalist and socialconstructivist. Characteristics such as originality of results and rigorous methodology are classified as universalist factors, and characteristics such as author's prestige and journal's prestige are classified as social-constructivist. With this perspective in mind, the research question of this dissertation is posed: what are factors that influence the citations received by a paper within the scenario of Brazilian accounting research? To answer this question, we collected data from 2,540 papers published in Brazilian accounting academic journals between 2007 and 2012. The data analysis is based on descriptive statistics, Pearson's correlation and chi-square test, correspondence analysis, and negative binomial regression. We created 16 hypotheses of factors that influenced the number of times articles were quoted. The rejected hypotheses were the ones about the influence of the number of terms in the title or in the keywords of the paper, the number of pages, the position of the article and the type of edition, the proportion of female authors, and the data analysis approach (qualitative or quantitative). The following hypotheses were only statistically significant in certain circumstances, and thus, were only partially accepted: the influence of the number of authors, the gender of the first author, the academic degree of the first author, the Qualis grade of the journal, and the age of the journal. The totally accepted hypotheses were the ones about the influence of the factors such as the institutional affiliation of the first author, the journal in which the paper was published, the research line, and the number of references in the article. From these results, we can state that, in Brazil, the citation pattern of a paper is influenced by social-constructivist criteria, but to a lesser degree than that of the international context, in which more socialconstructivist hypotheses are accepted. Therefore, in Brazil, the citation pattern is influenced by characteristics that are socially constructed and valued by the scientific community.
\end{abstract}

Keywords: Citation, Scientific journals, Scientific research, Accounting, Accounting research, Impact factor. 


\section{INTRODUÇÃO}

O avanço da ciência depende de dois processos interconectados, quais sejam o desenvolvimento de pesquisas e a disseminação dos resultados pela comunicação científica. Pesquisar e comunicar as descobertas são dois processos que interagem de forma tão intensa, atualmente, que podem parecer um só processo. Ou seja, muitas vezes, o processo de investigação é confundido com o produto dela, que são os artigos, perdendo-se, na discussão, a verdadeira natureza do processo de pesquisa. Para Meadows (1999, p. 85), "em termos de comunicação científica, as duas mais importantes características do pesquisador são a quantidade de informações que comunica e sua qualidade". Nesse sentido, a demanda por publicação se tornou tão grande que, na década de 1930, a academia já começava a apresentar críticas à máxima "Publish or perish".

A ciência da informação tem estudado os fenômenos relacionados à comunicação científica, observando que um dos mais interessantes é que pesquisas de cientistas famosos atraem, naturalmente, mais atenção do que pesquisas de cientistas não famosos. Esse fenômeno, batizado como efeito Mateus, foi descrito, inicialmente, por Merton (1968). Foi assim chamado em alusão ao versículo bíblico do evangelho de São Mateus: "Porque àquele que tem, se dará, e terá em abundância; mas àquele que não tem, até aquilo que tem Ihe será tirado" (Mateus, 13:12). O efeito Mateus caracteriza, ainda, a atribuição de citações a um artigo pela fama de seu autor e não, necessariamente, pelo mérito da pesquisa. llustração desse fenômeno é o caso de uma pesquisa que chama a atenção da academia poder ser indicada ao prêmio Nobel e, ao ser premiada, o prêmio atrai mais atenção ainda para a pesquisa. Law, Ye, Chen e Leung (2009) afirmam que o crescimento da importância da análise de citações sugere, fortemente, que o desempenho de pesquisa acadêmica está mudando, lentamente, o cenário de "Publish or Perish" para "Be cited or Vanish".

Segundo a literatura científica (Judge, Cable, Colbert e Rynes, 2007; Stremersch, Verniers, \& Verhoef, 2007), os artigos são citados por uma variada gama de razões, que podem ser classificadas entre dois polos: o polo universalista, que inclui razões, como inovação dos resultados, rigor metodológico, capacidade de explicação de um fenômeno; ou o polo socialconstrutivista, que abarca razões, como status dos autores, fama do periódico ou renome da instituição em que a pesquisa foi desenvolvida.

Partindo desse contexto, a questão de pesquisa neste artigo é: Quais fatores do polo social-construtivista influenciam o número de citações que um artigo sobre Contabilidade recebe no âmbito da pesquisa contábil brasileira? Assim, o objetivo desta pesquisa é identificar quais são os fatores que afetam o número de citações de um artigo no âmbito da pesquisa contábil brasileira, estando relacionado, portanto, com a maturidade da produção científica no país e com o impacto da pesquisa na área.

Para responder a questão de pesquisa exposta, fez-se uma análise de 2.540 artigos publicados por 36 periódicos científicos, entre os anos de 2007 e 2012, a fim de testar 16 hipóteses, por meio da análise de regressão binomial negativa, permitindo identificar que quatro variáveis estão relacionadas com o volume de 
"Diga-me Quantos te Citam, e Eu te Direi Quem és": Estudo Sobre as Citações no Âmbito da Pesquisa Contábil Brasileira

citações: afiliação institucional do primeiro autor; revista em que o artigo foi publicado; linha de pesquisa; e número de referências.

A justificativa desta pesquisa parte da necessidade de conhecer melhor como se comportam as citações dos artigos, de acordo as diferentes características que possuem. Identificar quais características do polo socialconstrutivista impactam o número de citações do artigo pode contribuir para que os autores reflitam sobre a sua prática científica e tomem decisões mais bem informados. Por outro lado, permite aos pesquisadores da área refletir sobre a maturidade da área e dos seus membros, a partir das escolhas que fazem.

No âmbito nacional, esta pesquisa se justifica, ainda, por apresentar originalidade, visto que, anterior a esta, uma única pesquisa foi realizada, analisando as citações dos artigos de periódicos de Contabilidade e relacionando-as com características dos artigos, que é o trabalho de Aragão, Oliveira e Lima (2014). Além disso, o Brasil apresenta características muito específicas que o distingue de outros países, como a tradição em manter todo o processo editorial livre de taxas, criando, inclusive, bases de dados de acesso livre, como a Scientific Electronic Library Online - Scielo e a Scientific Periodicals Electronic Library - Spell, distinguindo-se, ainda, de outros países por possuir um sistema próprio de avaliar a produção da pós-graduação, chamado Qualis.

Na sequência deste artigo, têm-se as seguintes partes: a fundamentação teórica, que posiciona a temática; a seção da metodologia, em que é apresentada a trajetória de pesquisa; a seção de análise de resultados, em que são discutidos os resultados da pesquisa, vis-à-vis com a literatura; e, finalmente, as conclusões, em que se resumem os achados, discutem-se as limitações e apontam-se possibilidades de pesquisas futuras.

\section{FUNDAMENTAÇÃO TEÓRICA}

Judge et al. (2007) afirmam que as publicações podem ser analisadas do ponto de vista universalista ou particularista da ciência. Na visão universalista, um artigo deve ser aceito para publicação quando: (1) oferece contribuições originais para a ciência; e (2) foi concebido e executado com elevado padrão de qualidade, independentemente da reputação ou colocação do autor no sistema de estratificação acadêmica. Já os céticos quanto a esse sistema acreditam numa visão particularista/social-construtivista, sugerindo que as citações podem basear-se na fonte da contribuição científica, como, por exemplo, a formação ou o status de um cientista, ao invés de seu conteúdo ou mérito.

\subsection{Pesquisas Relacionadas}

A literatura acerca do tema se amplia ao analisar outras áreas das ciências para além da área de negócios: no Direito, tem-se a pesquisa de Ayres e Vars (2000); em Ecologia, tem-se a pesquisa de Leimu e Koricheva (2005) e Fox, Paine e Sauterey (2016); em Marketing, tem-se a pesquisa de Stremersch et al. (2007) e Stremersch, Camacho, Vanneste e Verniers (2015); na área de Negócios, tem-se a pesquisa de Judge et al. (2007), Mingers e Xu (2010), Antonakis, Bastardoz, Liv e Schriesheim (2014); e, finalmente, em Contabilidade, tem-se a pesquisa de 
Martínez-Blasco, Argilés-Bosch, García-Blandón e Martínez de lbarreta Zorita (2016), na Espanha, e de Aragão et al. (2014), no Brasil. Cada um desses trabalhos de pesquisa serão, resumidamente, apresentados a seguir, em ordem cronológica.

Ayres e Vars (2000) investigaram o comportamento das citações de 979 artigos e outros documentos publicados em três revistas de Direito, entre os anos de 1980 e 1995. O estudo utilizou análise de dados em painel e identificou que a posição do artigo, no início, meio ou final da edição; a linha de pesquisa, o número de autores, a presença de figuras e de apêndice, a taxa de número de notas de rodapé por página, o sexo e a idade do(a) autor(a) impactam no número de citações. Afiliação institucional do autor e nepotismo dos artigos não impactam. Quando se incluem os outros documentos publicados pela revista, os fatores afiliação institucional, número de páginas e número de palavras no título (curtos) se tornam relevantes.

Leimu e Koricheva (2005) investigaram o comportamento das citações de 228 artigos publicados em 53 revistas de ecologia, entre os anos de 1975 e 2001. A coleta do número de citações foi realizada em janeiro de 2004. A análise foi feita por meio de correlações e testes de diferença de média. No artigo, identificou-se que impactam o número de citações: hipótese testada (se confirmação ou rejeição); número de páginas; número de autores; país e posição da instituição no ranking no Academic Ranking of World Universities; e revista. $O$ sexo do primeiro autor não impacta as citações.

Judge et al. (2007) investigaram o comportamento das citações de 614 artigos publicados em 21 revistas de negócios, entre os anos de 1990 e 1994. A análise foi realizada por meio de equações estruturais e identificou que afetam as citações: idade do artigo; tipo de artigo; número de referências; número de páginas; revista; posição do artigo na edição; afiliação dos autores e facilidade de leitura. Por outro lado, não afetam as citações: fração amostral; independência das fontes de dados; amostras não estudantis; método de pesquisa; sexo do primeiro autor; e confiabilidade.

Stremersch et al. (2007) investigaram o comportamento das citações de 1.825 artigos publicados em cinco revistas de marketing, entre os anos de $1990 \mathrm{e}$ 2002. A coleta do número de citações foi realizada em dezembro de 2004. A análise foi feita por meio de uma regressão binomial negativa e concluiu que as citações sofrem impacto de: posição do artigo na edição; premiação; número de páginas; tipo do artigo; linha de pesquisa; participação de autor no corpo editorial; posição da instituição no ranking; número de referências, de autocitação, de palavras no título; e presença de equações. Não impactam a quantidade de citações: número de publicações que os autores tinham nas cinco revistas; afiliação norte-americana; presença das palavras "Marketing", "Market", "New" no título; número de palavras-chave, de figuras, de tabelas, de notas de rodapé e de apêndices; facilidade de leitura; e revista.

Mingers e XU (2010) investigaram o comportamento das citações de 696 artigos publicados em seis revistas de gestão, no ano de 1990. A coleta do número de citações foi realizada em julho de 2008, no Web of Science (WoS). A análise foi feita por meio de regressão binomial negativa. Os percentuais de não citação foram de $4,5 \%$ a $22,7 \%$. O artigo registrou que há impacto das variáveis posição da instituição no ranking The Times Higher Education, número de referências de 
"Diga-me Quantos te Citam, e Eu te Direi Quem és": Estudo Sobre as Citações no Âmbito da Pesquisa Contábil Brasileira

páginas e de palavras-chave, tipo de artigo e revista. Constatou, ainda, que não há impacto das variáveis número de autores, de publicações do primeiro autor no WoS e de palavras no título, nacionalidade do primeiro autor e linha de pesquisa.

Antonakis et al. (2014) investigaram o comportamento das citações de 776 artigos sobre ciências sociais, publicados na The Leadership Quarterly, entre 1990 e 2012. A análise foi realizada por meio de uma regressão binomial negativa inflada de zeros e concluiu que há impacto das variáveis: tipo do artigo; número de referências, de autores e de artigos publicados pela revista por ano; média do número de citações anteriores recebidas pelos autores, do número de artigos prévios dos autores, do ranking da afiliação institucional dos autores; número da edição; linha de pesquisa; abordagem de análise dos dados; e desenho da pesquisa. Não há impacto de: idade do artigo; senioridade do editor; localização da amostra; número de fonte de dados; contexto temporal do estudo; e tipo de escala utilizada no número de citações.

Aragão et al. (2014) analisaram o comportamento das citações de 577 artigos sobre contabilidade, publicados em quatro revistas, entre os anos de 2006 e 2011. A análise foi realizada por meio de teste de qui-quadrado e por análise de correspondência. Os autores analisaram o impacto da revista, a idade do artigo e o idioma; e identificaram que são estatisticamente significantes.

Stremersch et al.(2015) investigaram o comportamento das citações de 659 artigos de cinco revistas de marketing, entre os anos de 1990 e 2007. A análise foi feita por meio de regressão binomial negativa. $O$ artigo identificou uma influência estatisticamente significativa de: premiação; linha de pesquisa; número de publicações que os autores tinham nas cinco revistas e de autocitação; e facilidade de leitura. Muitos fatores não tiveram impacto estatístico significante: posição do artigo na edição; número de páginas; tipo do artigo; participação no corpo editorial; ranking da instituição no Financial Times; centralidade; país; número de autores, de referências, de palavras no título, de palavras-chave, de equações, de figuras, de tabelas, de notas de rodapé, de apêndices; e presença das palavras "Marketing", "Market" e "New" no título.

Fox et al. (2016) analisaram o comportamento das citações de 26.088 artigos publicados por 32 revistas de ecologia, entre 2009 e 2012. A coleta das citações ocorreu em outubro de 2014 por meio da Web of Science. Os autores analisaram o impacto de fatores, como número de referências, de páginas, e de autores, concluindo que esses fatores são estatisticamente significantes.

Martínez-Blasco et al. (2016) analisaram o comportamento das citações dos 134 artigos publicados no Spanish Journal of Finance and Accounting, entre os anos de 2008 e 2013. A análise foi realizada por meio de regressão de Poisson. Os autores identificaram que as citações sofrem impacto do número de páginas, de autores, de publicações anteriores dos autores, do tipo de edição, do idioma, e da localização da amostra. Não sofrem impacto da posição na edição, do número de referências, da participação do autor no conselho editorial, da idade do artigo, do método de pesquisa, do tamanho da amostra e de participação no mainstream.

O levantamento dessas pesquisas, realizadas anteriormente, permitiu a construção da maioria das hipóteses testadas nesta pesquisa, como as hipóteses 
acerca da quantidade de autores por artigo e o sexo do primeiro autor. A seguir, são relacionadas as 16 hipóteses e a fundamentação teórica de cada uma delas.

Hipóteses relacionadas à autoria do artigo:

\begin{tabular}{|c|c|}
\hline Hipótese & Fundamentação teórica \\
\hline $\begin{array}{l}\text { H1: A quantidade de autores impacta a } \\
\text { quantidade de citações do artigo }\end{array}$ & $\begin{array}{l}\text { Ayres \& Vars, 2000; Leimu \& Koricheva, 2005; } \\
\text { Antonakis et al. 2014; Martínez-Blasco, } \\
\text { Argilés-Bosch, García-Blandón, \& Zorita, } \\
\text { 2016; Fox et al., } 2016\end{array}$ \\
\hline $\begin{array}{l}\text { H2: O gênero do primeiro autor impacta a } \\
\text { quantidade de citações do artigo }\end{array}$ & Ayres \& Vars, 2000 \\
\hline $\begin{array}{l}\text { H3: A proporção de autoras impacta a } \\
\text { quantidade de citações do artigo }\end{array}$ & Autoria própria \\
\hline $\begin{array}{l}\text { H4: A titulação do primeiro autor impacta a } \\
\text { quantidade de citações do artigo }\end{array}$ & Ayres \& Vars, 2000 \\
\hline $\begin{array}{l}\text { H5: A afiliação institucional do primeiro autor } \\
\text { impacta a quantidade de citações do artigo }\end{array}$ & $\begin{array}{l}\text { Ayres \& Vars, 2000; Leimu \& Koricheva, 2005; } \\
\text { Stremersch et al., 2007; Mingers \& Xu, } 2010\end{array}$ \\
\hline$x$ & : \\
\hline Hipótese & Fundamentação teórica \\
\hline $\begin{array}{l}\text { H6: A revista impacta a quantidade de } \\
\text { citações do artigo }\end{array}$ & $\begin{array}{l}\text { Leimu \& Koricheva, 2005; Judge et al., 2007; } \\
\text { Mingers \& Xu, 2010; Aragão et al., } 2014\end{array}$ \\
\hline $\begin{array}{l}\text { H7: O tipo de edição impacta a quantidade } \\
\text { de citações do artigo }\end{array}$ & Martínez-Blasco et al., 2016 \\
\hline $\begin{array}{l}\text { H8: O estrato do Qualis da revista impacta a } \\
\text { quantidade de citações do artigo }\end{array}$ & Autoria própria \\
\hline $\begin{array}{l}\text { H9: A idade da revista impacta a quantidade } \\
\text { de citações do artigo }\end{array}$ & Autoria própria \\
\hline $\begin{array}{l}\text { H10: A posição do artigo na edição impacta } \\
\text { a quantidade de citações do artigo }\end{array}$ & $\begin{array}{l}\text { Ayres \& Vars, 2000; Judge et al., 2007; } \\
\text { Stremersch et al., } 2007\end{array}$ \\
\hline
\end{tabular}

Hipóteses relacionadas à característica do artigo:

\begin{tabular}{|c|c|}
\hline Hipótese & Fundamentação teórica \\
\hline $\begin{array}{l}\text { H1 1: O número de termos no título impacta a } \\
\text { quantidade de citações do artigo }\end{array}$ & Ayres \& Vars, 2000 \\
\hline $\begin{array}{l}\text { H12: O número de termos nas palavras- } \\
\text { chave impacta a quantidade de citações } \\
\text { do artigo }\end{array}$ & $\begin{array}{l}\text { Stremersch et al., 2007; Mingers \& Xu, 2010; } \\
\text { Stremersch, Camacho, Vanneste, \& } \\
\text { Verniers, 2015 }\end{array}$ \\
\hline $\begin{array}{l}\text { H13: O número de páginas impacta a } \\
\text { quantidade de citações do artigo }\end{array}$ & $\begin{array}{l}\text { Ayres \& Vars, 2000; Leimu \& Koricheva, 2005; } \\
\text { Judge et al., 2007; Stremersch et al., 2007; } \\
\text { Mingers \& Xu, 2010; Fox et al., } 2016\end{array}$ \\
\hline $\begin{array}{l}\text { H14: O número de referências impacta a } \\
\text { quantidade de citações do artigo }\end{array}$ & $\begin{array}{l}\text { Judge et al., 2007; Stremersch et al., 2007; } \\
\text { Mingers \& Xu, 2010; Antonakis et al., 2014; Fox } \\
\text { et al., } 2016\end{array}$ \\
\hline $\begin{array}{l}\text { H15: A linha de pesquisa do artigo impacta a } \\
\text { quantidade de citações do artigo }\end{array}$ & $\begin{array}{l}\text { Ayres \& Vars, 2000; Stremersch et al., 2007; } \\
\text { Antonakis et al., 2014; Stremersch et al., } 2015\end{array}$ \\
\hline $\begin{array}{l}\text { H16: A abordagem da análise de dados } \\
\text { impacta a quantidade de citações do artigo }\end{array}$ & Antonakis et al., 2014 \\
\hline
\end{tabular}

A Hl surge ao se refletir sobre o papel do autor em difundir seu artigo a potenciais novos autores citantes, ou sobre o papel do autor ao dar continuidade 154 Revista Contabilidade Vista \& Revista, ISSN 0103-734X, Universidade Federal de Minas Gerais, Belo Horizonte, v. 31, n. 3, p. .148-169, set./dez. 2020 
"Diga-me Quantos te Citam, e Eu te Direi Quem és": Estudo Sobre as Citações no Âmbito da Pesquisa Contábil Brasileira

em sua agenda de pesquisa e reportar, nos artigos futuros, os achados que já descobriu em pesquisas anteriores. Assim, quanto mais autores tiver um artigo, mais chances ele tem de ser exposto a um público maior ou de ser mencionado em artigos futuros pelos próprios autores (autocitação). A H2 surge porque as pesquisas de autoria feminina, no passado, quando as mulheres tinham menor participação na academia, tendiam a ser menos citadas que as de autoria masculina, com exceção de áreas como enfermagem e serviço social, por exemplo. A H3 é apenas uma extensão da análise da variável sexo do primeiro autor. A H4 surge da expectativa criada acerca da qualidade do artigo em função do grau de experiência em pesquisa científica de quem a conduz, que, normalmente, ocupa o lugar de primeiro autor nos artigos da área de Administração e Contabilidade, no Brasil. A H5 surge do fato de que autores pegam emprestada a credibilidade das IES quando sinalizam à comunidade científica a sua afiliação institucional.

A H6 surge pela imagem que o periódico tem perante a comunidade científica. A H7 surge pelo fato de que as edições especiais recebem um tratamento especial, que as distingue do processo editorial normal, às vezes sendo objeto de ações de marketing por parte dos periódicos, o que poderia atrair mais atenção para os artigos publicados nessas edições do que nas edições ordinárias. A H8, acerca do impacto do Qualis da revista no volume das citações, não estava prevista nas pesquisas anteriores, pois o Qualis é um sistema brasileiro de avaliação, não utilizado em outros países, mas foi incluída por se entender que receber uma classificação em determinado estrato pode influenciar ter seus artigos mais ou menos citados. A H9, sobre idade da revista, também foi incluída ao se constatar que a maioria das revistas brasileiras são relativamente jovens, algumas, inclusive, foram criadas durante o período analisado, como se detalhará na próxima seção. A H10 surge em função do fenômeno notado por Simon Newcomb de que as primeiras páginas dos livros pareciam ser muito mais usadas que as últimas, percepção que levaria, no futuro, a se elaborar a Lei Newcomb-Benford. De maneira análoga, os artigos publicados como o primeiro da edição receberiam mais atenção que os demais.

As $\mathrm{H} 11$ e H12 surgem do fato de que a probabilidade de artigos que têm mais descritores serem recuperados em mecanismos de busca booleana, ceteris paribus, é maior que de artigos que têm menos descritores. A H13 surge considerando que artigos mais longos tiveram mais espaço para detalhar seu conteúdo, podendo, assim, serem melhor compreendidos pelos leitores. A H14 surge porque um volume maior de referências pode indicar um volume maior de literatura revisada, indicando maior robustez da fundamentação e maior cotejamento de resultados com a literatura. A H15 deriva de que o volume de pesquisadores que atuam em cada área de pesquisa é distinto, e essa variação do volume de pesquisadores pode aumentar ou diminuir as citações dos artigos de áreas distintas. Por fim, a H16 surge da tradição da área em usar abordagens quantitativas, muitas delas, econométricas.

Na próxima seção, são detalhados os procedimentos metodológicos desenvolvidos para testar as hipóteses da pesquisa. 


\section{PROCEDIMENTOS METODOLÓGICOS}

Esta pesquisa se baseia na análise das citações dos artigos publicados por revistas brasileiras de contabilidade e controladoria, nos triênios de 2007-2009 e 2010-2012. Foram incluídas as revistas constantes da lista publicada nos sítios eletrônicos da Associação Nacional dos Programas de Pós-Graduação em Contabilidade (Anpcont) e do Núcleo de Estudos sobre Meio Ambiente e Contabilidade da Universidade Federal de Santa Catarina, no início de 2013.

A Figura 1 elenca as revistas da amostra desta pesquisa, ordenadas pelo ano de criação, apresentado na terceira coluna.

\begin{tabular}{|c|c|c|c|}
\hline Título da revista & Instituição & Ano & Sigla da revista \\
\hline Revista de Contabilidade \& Finanças & USP & 1989 & $\mathrm{RC} \& \mathrm{~F}$ \\
\hline Contabilidade Vista \& Revista & UFMG & 1989 & CVR \\
\hline Contabilidade, Gestão e Governança & UnB & 1998 & CGG \\
\hline Revista Brasileira de Contabilidade & CFC & 1999 & RBC \\
\hline Revista Catarinense da Ciência Contábil & CRC SC & 2001 & Catarinense_CC \\
\hline ConTexto & UFRGS & 2001 & Contexto \\
\hline $\begin{array}{l}\text { Revista de Contabilidade do Mestrado em } \\
\text { Ciências Contábeis }\end{array}$ & UERJ & 2003 & RCMCC \\
\hline Contextus & UFC & 2003 & Contextus \\
\hline Revista Eletrônica de Contabilidade & UFSM & 2004 & R_Elet_Cont \\
\hline Revista Contemporânea de Contabilidade & UFSC & 2004 & RCC_UFSC \\
\hline Pensar Contábil & CRC RJ & 2004 & Pensar \\
\hline Brazilian Business Review & FUCAPE & 2004 & BBR \\
\hline BASE & UNISINOS & 2004 & Base \\
\hline Revista Universo Contábil & FURB & 2005 & RUC \\
\hline Revista Brasileira de Gestão de Negócios & FECAP & 2005 & RBGN \\
\hline Enfoque: Reflexão Contábil & UEM & 2005 & Enfoque \\
\hline Custos e @gronegócio online & UFRPE & 2005 & Custos_e_@agro \\
\hline Sociedade, Contabilidade e Gestão & UFRJ & 2006 & SCG \\
\hline CAP Accounting and Management & UTFPR & 2006 & CAP \\
\hline ABCustos & $A B C$ & 2006 & ABCustos \\
\hline Revista de Informação Contábil & UFPE & 2007 & RIC \\
\hline Revista de Educação e Pesquisa em Contabilidade & CFC & 2007 & REPEC \\
\hline Revista de Contabilidade e Organizações & USP/RP & 2007 & RCO \\
\hline Revista de Contabilidade da UFBA & UFBA & 2007 & RC_UFBA \\
\hline $\begin{array}{l}\text { RACE: Revista de Administração, Contabilidade e } \\
\text { Economia }\end{array}$ & UNOESC & 2007 & RACE \\
\hline Advances in Scientific and Applied Accounting & ANPCONT & 2008 & ASAA \\
\hline Revista de Contabilidade e Controladoria & UFPR & 2009 & RCC_UFPR \\
\hline Revista de Administração e Contabilidade da FAT & FAT & 2009 & RAC_FAT \\
\hline Revista Ambiente Contábil & UFRN & 2009 & Amb_Cont \\
\hline Revista de Estudos Contábeis & UEL & 2010 & R_Est_Cont \\
\hline Registro Contábil & UFAL & 2010 & Recont \\
\hline $\begin{array}{l}\text { RACEF - Revista de Administração, Contabilidade e } \\
\text { Economia da FUNDACE }\end{array}$ & FUNDACE & 2010 & RACEF \\
\hline Revista de Gestão, Finanças e Contabilidade & UNEB & 2011 & RGFC \\
\hline $\begin{array}{l}\text { Reunir: Revista de Administração, Contabilidade e } \\
\text { Sustentabilidade }\end{array}$ & UFCG & 2011 & Reunir \\
\hline Revista Razão Contábil \& Finanças & FATE & 2012 & Razao \\
\hline REAVI - Revista Eletrônica do Alto Vale do Itajaí & UDESC & 2012 & REAVI \\
\hline
\end{tabular}

Figura 1. Revistas que compuseram a amostra

Fonte: Elaborada pelos autores 
"Diga-me Quantos te Citam, e Eu te Direi Quem és": Estudo Sobre as Citações no Âmbito da Pesquisa Contábil Brasileira

Analisando a Figura 1, constata-se que as primeiras revistas publicadas na área, no Brasil, datam de 1989 e são a Revista de Contabilidade \& Finanças, mantida pela Universidade de São Paulo (USP), e a revista Contabilidade Vista \& Revista, editada pela Universidade Federal de Minas Gerais (UFMG). A Revista Brasileira de Contabilidade, embora tenha sido criada em 1912, aparece como criada em 1999, pois é nesse ano que as primeiras edições constavam online, no sítio da revista. Apesar desse início lento, houve uma ampla expansão no número de revistas na área, principalmente, a partir do novo milênio.

\subsection{Coleta de Dados}

Os artigos foram coletados no sítio de cada revista e baixados em formato Portable Document Formatß. As informações de cada artigo foram, manualmente coletadas e organizadas em uma planilha. A amostra inicial era composta por três mil artigos, da qual, depois de excluídos registros com dados faltantes (missing data), formou-se a amostra final, composta de 2.540 artigos.

As variáveis utilizadas são classificadas em quantitativas ou qualitativas. Entre as variáveis quantitativas estão: número de termos no título do artigo, nas palavras-chave do artigo, de páginas, de referências, de autores; proporção de autoras; idade da revista no ano em que o artigo foi publicado; e idade do artigo. As variáveis qualitativas, por sua vez, são: revista; tipo de edição; posição do artigo; sexo do primeiro autor; titulação acadêmica do primeiro autor; instituição do primeiro autor; linha de pesquisa; estrato do Qualis vigente no ano em que o artigo foi publicado; e abordagem na análise de dados. A forma de obtenção de cada um desses fatores é, a seguir, brevemente explicada, sendo cada uma delas detalhada na sequência.

O número de termos no título do artigo e o número de termos nas palavraschave do artigo foram identificados com o uso do aplicativo online Webxtool®, que fez a contagem das palavras. Os valores possíveis dessas variáveis, teoricamente, eram de $1 a+\infty$. No entanto, como a Revista Brasileira de Contabilidade não utiliza palavras-chave, resultou a ocorrência de dados faltantes. Assim, a média de termos nas palavras-chave das demais 35 revistas foi utilizada para preencher os dados faltantes dessa revista. A média nas palavraschave de cada artigo era de sete termos.

O número de páginas foi recuperado nos artigos e teve valores possíveis de $1 a+\infty$. O número de autores total e o número de referências foram obtidos por meio de contagem. Os valores possíveis para o número de autores eram de $1 \mathrm{a}+$ $\infty$. Já os valores possíveis para o número de referências eram de $0 a+\infty$, porque, embora não seja usual, um artigo não precisa, necessariamente, ter alguma referência. De fato, houve a ocorrência de um artigo que não possuía nenhuma referência.

A proporção de autoras foi obtida por meio da contagem de autoras mulheres, dividido esse valor pelo número total de autores do artigo. Considerando que o número de autores variava de 1 a 6 e que poderia haver diferentes quantidades de autoras mulheres, os possíveis valores para essa variável eram $0 ; 0,16 ; 0,20 ; 0,25 ; 0,33 ; 0,40 ; 0,50 ; 0,60 ; 0,66 ; 0,75 ; 0,80 ; 0,84$ e 1. 
A idade da revista foi calculada com base no ano da mais antiga edição online disponível da revista, mesmo que tenha havido mudança de nome do periódico no período, subtraída do ano de publicação do artigo. Assim, a idade da revista, registrada para um artigo publicado na Revista Contabilidade \& Finanças, em 2007, levou em consideração não apenas o período de 2001 a 2007, em que a revista possuía essa denominação, mas também os 12 anos de existência com a denominação de Caderno de Estudos. Os valores possíveis para essa variável eram de 0 a 23, que eram os artigos publicados no mesmo ano que a revista foi criada até os artigos publicados, em 2012, na Revista Contabilidade \& Finanças e na Contabilidade Vista \& Revista.

Já a idade do artigo foi calculada subtraindo o ano em que o artigo foi publicado do ano de 2016, que foi o ano da coleta dos dados usados nas análises. Os possíveis valores eram de 4 a 9, que eram os artigos publicados de 2007 a 2012. A idade da revista e a idade do artigo foram calculadas, automaticamente, com o uso de funções pelo Microsoft Excelß.

Os artigos são publicados em dois tipos de edição, que se denominou de ordinária e especial. As edições ordinárias consistem nas edições que seguem o processo normal de editoração e avaliação das revistas. As edições especiais são as edições em que pode haver alterações do processo normal de editoração, como a editoria de um editor convidado ou a chamada de artigos para um tema específico.

A posição dos artigos dentro da edição foi operacionalizada classificando cada artigo apresentado, sendo o primeiro da lista da edição classificado como "Primeiro", e os demais como "Outro".

A classificação do sexo do primeiro autor foi feita em etapas. Inicialmente, a classificação foi feita por meio da leitura do nome do primeiro autor. Nomes brasileiros comuns, como José e Maria, foram de fácil classificação. O problema foi a classificação de nomes andrógenos, como Joisse e Sidney, e nomes estrangeiros, como Jiri, por exemplo. Nesses casos, procedeu-se a uma busca na Plataforma Lattes pelo nome completo do(a) autor(a). Quando não havia currículo cadastrado na Plataforma Lattes, procedeu-se à busca no Google. E, quando não foi possível identificar com segurança o sexo no autor, a observação foi eliminada da amostra. O sexo foi dividido entre masculino e feminino.

A identificação da titulação do primeiro autor também foi feita em etapas, pois, embora a grande maioria das revistas identifique essa informação logo após o nome do autor, nem todas o fazem. Nesses casos, procedeu-se a uma busca pelo currículo Lattes do(a) autor(a) para identificar a titulação que ele(a) possuía no ano de publicação do artigo. Quando a Plataforma Lattes não esclarecia esse ponto, procedeu-se a buscas no Google. Quando a busca não era suficiente para o esclarecimento, a observação foi eliminada.

Há que se registrar que uma reclassificação foi feita com a seguinte diretriz: autores que já concluíram o doutorado foram reclassificados como "doutor" (incluíram-se aqui as categorias doutor, pós-doutorando, pós-doutor, livredocente, professor titular); autores que já concluíram o mestrado, mas não o doutorado, foram reclassificados como "mestre" (incluindo-se aqui as categorias mestre, doutorando, aluno de doutorado); autores que já concluíram a graduação, mas não o mestrado, foram reclassificados como "bacharel" (incluíram-se aqui as categorias bacharel, contador, administrador, especialista, 
"Diga-me Quantos te Citam, e Eu te Direi Quem és": Estudo Sobre as Citações no Âmbito da Pesquisa Contábil Brasileira

especializando, aluno de especialização, aluno de pós-graduação, mestrando, aluno de mestrado); e os alunos que não concluíram a graduação foram classificados como "graduando".

Para a instituição de ensino superior do primeiro autor utilizou-se, inicialmente, a instituição declarada pelo(a) autor(a) no artigo. Um problema encontrado nessa variável foi que alguns autores indicavam duas ou mais instituições, normalmente aquela em que cursaram o doutorado e/ou mestrado e aquela em que atuavam como docentes (instituição de vínculo). De fato, isso aconteceu com maior frequência numérica com quem fez doutorado na USP e atuava em outras instituições. Diante disso, a opção adotada na pesquisa foi marcar a primeira instituição indicada pelo(a) autor(a), visto que a alternativa seria separar a instituição de formação da instituição de atuação. Além disso, houve os casos em que não identificavam o tipo de vínculo, casos em que só indicavam o vínculo de formação e casos em que só havia indicação do vínculo de ałuação, de modo que a amostra seria subdividida em subgrupos numericamente inferiores.

Sobre a afiliação institucional, quando a instituição indicada possuía um programa de pós-graduação stricto sensu em Contabilidade, a marcação foi feita com o nome da instituição. Quando não havia essa informação, a marcação foi feita em uma categoria agrupada, denominada "Outro". Umm fato incomum observado foi a identificação que alguns autores fizeram de sua afiliação institucional com o Programa Multi-Institucional e Inter-regional de PósGraduação em Ciências Contábeis da UnB/UFPB/UFRN. Esses casos foram classificados como pertencentes à categoria UnB.

Sobre a classificação das revistas brasileiras de Contabilidade no Qualis, vigente nos triênios de 2007-2009 e 2010-2012, foi possível distribuí-las em oito categorias: A2, B1, B2, B3, B4, B5, C e NC (Não classificado). Das 36 revistas da amostra, apenas sete (RACEF, REAVI, RGFC, Razão Contábil, Recont, Reunir e Revista de Estudos Contábeis) foram classificadas em apenas um estrato ao longo do período analisado. Nesses sete casos, isso ocorreu porque elas não existiam no triênio 2007-2009. As demais revistas tiveram mais de uma classificação durante o perío do analisado, sendo isso, também, levado em consideração nas análises.

Quanto à abordagem de pesquisa, foi dividida entre abordagem quantitativa e não quantitativa. A escolha por essa classificação deveu-se à hipótese de que o emprego de métodos quantitativos na análise dos dados impacta, positivamente, a citação. A abordagem foi identificada nas informações dadas pelos artigos. A quase totalidade dos artigos da amostra classificava a pesquisa em exploratória, descritiva ou explicativa. Na sequência, a maioria dos artigos identificava a técnica empregada na análise dos dados como, por exemplo, análise de regressão, análise de séries temporais, etnografia, grounded theory, análise de conteúdo, análise do discurso, entre outras. Essas foram as técnicas marcadas na planilha e que, posteriormente, foram categorizadas entre pesquisas quantitativas ou não quantitativas. Todos os métodos quantitativos, como regressões, análise de agrupamentos, análise de correspondência, testes de hipóteses e similares, foram tratados como abordagem quantitativa. Análise de conteúdo, estudos de caso, análise de discurso e similares foram classificadas como abordagem não quantitativa. 
Por opção metodológica, pesquisas que se ativeram ao emprego de estatísticas descritivas, como mínimo, máximo, moda; e medidas-resumo, como média, desvio-padrão, variância e percentual, foram classificadas como abordagem não quantitativa. Tomou-se essa medida visto que pesquisas qualitativas podem empregar essas informações e, ainda assim, a análise permanecer essencialmente qualitativa.

A coleta no número de citações utilizadas deu-se por meio de consulta realizada ao Google Acadêmico, durante o mês de abril de 2016. No entanto, três outras coletas exploratórias já haviam sido realizadas em abril de 2014, abril de 2015 e julho de 2015 para verificar a confiabilidade do buscador. As duas coletas iniciais, inclusive, comportavam apenas as revistas mantidas por programas de pós-graduação em controladoria e contabilidade. A consulta utilizou o software Harzing Publish or Perish® (Harzing, 2007).

\subsection{Estratégia de Análise de Dados}

A regressão binomial negativa foi utilizada para identificar o impacto dos fatores (variáveis explicativas) no número de citações dos artigos. A regressão binomial negativa também havia sido usada pelos estudos de Stremersch et al. (2007), Mingers e Xu (2010) e Antonakis et al. (2014). O procedimento stepwise foi empregado para eliminar as variáveis e dummies que não fossem estatisticamente significantes em um nível de significância de 5\%. A taxa de incidência estimada (irr - incidence rate ratio) foi apresentada em percentual em todas as variáveis que sobreviveram ao procedimento stepwise.

Fávero (2015, p. 198) explica que os modelos de regressão binomial negativa "são enquadrados nos chamados modelos de regressão de dados de contagem, sendo apropriados para estimação quando a variável dependente for quantitativa e com valores inteiros e não negativos [...] e quando houver superdispersão dos dados". Para Fávero, Belfiori, Silva, \& Chan (2009, p. 365), o procedimento stepwise é, frequentemente, utilizado quando "o pesquisador deseja avaliar a significância estatística dos parâmetros de determinadas variáveis explicativas por meio da inclusão passo a passo apenas daquelas que se mostraram relevantes a determinado nível de confiança [...] e é bastante útil quando o pesquisador quer considerar um número grande de variáveis explicativas". Fávero (2015) explica, ainda, que a irr permite identificar o efeito, em percentual, de quanto se altera uma variável explicativa em uma unidade ou com a presença ou não de uma categoria qualitativa.

Em resumo, foi realizada uma regressão binomial negativa com uma amostra de 2.540 observações com o uso do software Stata, versão 14, seguindo as rotinas propostas por Fávero (2015). A variável dependente utilizada foi a quantidade de citações que cada artigo recebeu, e as variáveis independentes foram as sete variáveis quantitativas e as nove variáveis qualitativas descritas anteriormente. Considerando as características dos dados, quando foi implementada a regressão no Stataß, optou-se por utilizar o procedimento stepwise para eliminar as variáveis, ou dummies que não fossem estatisticamente diferentes de zero. O nível de significância adotado foi de $5 \%$. 
"Diga-me Quantos te Citam, e Eu te Direi Quem és": Estudo Sobre as Citações no Âmbito da Pesquisa Contábil Brasileira

\section{ANÁLISE DOS RESULTADOS}

\subsection{Análise dos Modelos Elaborados}

Foram elaborados oito modelos. A eficácia de cada modelo foi avaliada considerando-se o Pseudo r2 de McFadden, conforme consta da Tabela 1.

\begin{tabular}{lll} 
Tabela 1 & & \\
Pseudo R2 & de McFadden dos modelos alternativos gerados na pesquisa & \\
\hline Modelo & Característica & Pseudo R2 de McFadden \\
\hline Modelo 1 & com todas as variáveis & 0,0677 \\
\hline Modelo 2 & sem a variável Revista & 0,0318 \\
\hline Modelo 3 & sem a variável ldade da revista & 0,0641 \\
\hline Modelo 4 & $\begin{array}{l}\text { sem as variáveis Idade da revista e } \\
\text { Qualis }\end{array}$ & 0,0554 \\
\hline Modelo 5 & com a variável ldade do artigo & 0,0669 \\
\hline Modelo 6 & $\begin{array}{l}\text { sem a USP como categoria de IES } \\
\text { do primeiro autor }\end{array}$ & 0,0645 \\
\hline Modelo 7 & $\begin{array}{l}\text { sem a RC\&F como categoria de } \\
\text { Revista }\end{array}$ & 0,0488 \\
\hline Modelo 8 & $\begin{array}{l}\text { sem a USP como categoria de IES } \\
\text { do primeiro autor e sem a RC\&F } \\
\text { como categoria de Revista }\end{array}$ & 0,0446 \\
\hline
\end{tabular}

Fonte: Elaborada pelos autores

$O$ pseudo $\mathrm{R}^{2}$ de McFadden de todos os modelos gerados com menos variáveis ou categorias dummy foi menor. Ressalva-se que, embora o Modelo 5 tenha um pseudo $\mathrm{R}^{2}$ de McFadden 0,0008, menor que o Modelo 1, apresentou taxas de incidência estimadas mais em conformidade com a estatística descritiva das variáveis analisadas, principalmente para as Revista Contabilidade \& Finanças e revista Contabilidade Vista \& Revista.

A categoria Contabilidade Gerencial e Controladoria, do fator linha de pesquisa, apareceu em todos os modelos, enquanto que a categoria Mercado de Capitais apareceu nos Modelos 1, 3, 5, 6 e 7. Todos os modelos indicaram que os artigos dessas duas linhas são, em média, menos citados que da linha Educação e Pesquisa em Contabilidade, que é a categoria de referência.

O sexo do primeiro autor mostrou-se estatisticamente significante em seis modelos, não aparecendo, apenas, nos Modelos 2 e 6. Os modelos nos quais apareceu indicam que os artigos com primeira autora feminina são mais citados do que com primeiro autor masculino. Esse resultado é surpreendente quando considerada a literatura. As categorias UFPB e Outro, da instituição do ensino do primeiro autor, apareceram em todos os modelos. A USP apareceu em seis modelos; a Fucape, no Modelo 3; e a UnB e a FURB, no Modelo 4.

A variável revista apareceu em todos os sete modelos nos quais foi incluída. O Modelo 5 foi o que reteve menos categorias (14 revistas) e o Modelo 1 reteve mais categorias (27 revistas). Aragão et al. (2014), em um estudo com quatro revistas brasileiras de contabilidade, já haviam detectado que a revista influencia o número de citações. Com esta pesquisa, pode-se afirmar que essa influência é exercida por, pelo menos, outros 14 periódicos. 
A idade da revista foi incluída em seis modelos, mostrando-se estatisticamente significante em cinco desses modelos. Somente no Modelo 5 foi incluída na regressão, mas excluída pelo procedimento stepwise. As categorias Doutor e Mestre, da titulação acadêmica do primeiro autor, mostraram-se significantes no Modelo 2. A abordagem quantitativa de análise de dados mostrou-se significante somente no Modelo 4.

A variável número de autores mostrou-se estatisticamente significativa somente no Modelo 5. As categorias Bl e B3, do estrato Qualis da revista, mostraram-se significantes nos Modelos 2 e 5. A categoria B2 mostrou-se significante no Modelo 7. Todos os estratos Qualis mostraram-se significantes no Modelo 3.

A única variável explicativa quantitativa que se mostrou estatisticamente significativa em todos os modelos foi o número de referências, sempre no sentido positivo e na proporção entre $0,5 \%$ a $1,2 \%$. As variáveis recorrentes em todos os modelos gerados permitiram aceitar as hipóteses de influência: afiliação institucional, revista, número de referências, e linha de pesquisa, acarretando a aceitação das hipóteses H5, H6, H14 e H15.

Por último, a idade do artigo, que segundo Aragão et al. (2014) influencia as citações, acabou por se mostrar estatisticamente significante no Modelo 5. Esse resultado era esperado pois há uma tendência de crescimento do número de citações ao longo do período estudado.

\subsection{Análise dos Resultados do Melhor Modelo}

Detalham-se, a seguir, os resultados do melhor modelo gerado na pesquisa, o modelo com todos os fatores incluídos, conforme apresentado na Tabela 2. 
"Diga-me Quantos te Citam, e Eu te Direi Quem és": Estudo Sobre as Citações no Âmbito da Pesquisa Contábil Brasileira

\section{Tabela 2}

Resultados do melhor modelo gerado na pesquisa

\begin{tabular}{l|l}
\hline Number of obs $=2.540$ & Prob $>$ chi2 $=0,0000$ \\
\hline LR chi2 $(35)=895,81$ & Log likelihood $=-6163,8131$ \\
\hline Dispersion $=$ mean & Pseudo R2 $=0,0677$ \\
\hline
\end{tabular}

\begin{tabular}{lccc}
\hline Citações & IRR & Std. Err. & P $\mathbf{z}$ \\
\hline Constante & 5,35 & 0,50 & 0,00 \\
\hline Gerencial e Controladoria & 0,69 & 0,04 & 0,00 \\
\hline Mercado de Capitais & 0,84 & 0,06 & 0,01 \\
\hline Mulher & 1,14 & 0,06 & 0,01 \\
\hline UFPB & 1,67 & 0,39 & 0,03 \\
\hline USP & 1,34 & 0,10 & 0,00 \\
\hline Outro & 0,81 & 0,05 & 0,00 \\
\hline Contextus & 2,51 & 0,58 & 0,00 \\
\hline Pensar & 1,36 & 0,18 & 0,02 \\
\hline RACEF & 0,19 & 0,07 & 0,00 \\
\hline RAC_FAT & 0,16 & 0,06 & 0,00 \\
\hline RBC & 2,99 & 0,47 & 0,00 \\
\hline RBGN & 2,51 & 0,42 & 0,00 \\
\hline RC\&F & 125,73 & 30,64 & 0,00 \\
\hline RCC_UFPR & 0,58 & 0,09 & 0,00 \\
\hline RCC_UFSC & 2,47 & 0,33 & 0,00 \\
\hline RCMCC & 1,86 & 0,26 & 0,00 \\
\hline RCO & 1,68 & 0,20 & 0,00 \\
\hline RC_UFBA & 0,63 & 0,10 & 0,00 \\
\hline REAVI & 0,19 & 0,14 & 0,02 \\
\hline REPEC & 1,53 & 0,19 & 0,00 \\
\hline RGFC & 0,56 & 0,15 & 0,03 \\
\hline RUC & 1,89 & 0,19 & 0,00 \\
\hline Amb_Cont & 0,39 & 0,08 & 0,00 \\
\hline R_Elet_Cont & 0,11 & 0,11 & 0,04 \\
\hline R_Est_Cont & 0,24 & 0,07 & 0,00 \\
\hline Razao & 0,29 & 0,09 & 0,00 \\
\hline Recont & 0,24 & 0,07 & 0,00 \\
\hline Reunir & 0,33 & 0,09 & 0,00 \\
\hline BBR & 2,32 & 0,35 & 0,00 \\
\hline Base & 2,06 & 0,28 & 0,00 \\
\hline CGG & 6,82 & 1,11 & 0,00 \\
\hline CVR & 46,18 & 11,37 & 0,00 \\
\hline Contexto & 3,08 & 0,50 & 0,00 \\
\hline Idade & 0,83 & 0,01 & 0,00 \\
\hline Número de referências & 1,01 & 0,00 & 0,00 \\
\hline Ront_:Eaborada & & & \\
\hline
\end{tabular}

Fonte: Elaborada pelos autores

Sobre os dados da Tabela 2, cabe esclarecer que, quando as variáveis dummies foram criadas, a área de pesquisa omitida foi a de Educação e Pesquisa em Contabilidade. As áreas de Contabilidade Gerencial e Controladoria e de Mercado de Capitais mantiveram-se no modelo após a aplicação do procedimento stepwise. A área de Contabilidade Gerencial e Controladoria teve um irr =0,69; e a área de Mercado de Capitais, um irr =0,83. Isso significa que, mantidas constantes as demais condições, um artigo pertencente à linha de Contabilidade Gerencial e Controladoria teve 31\% (1-0,69=0,31) menos citações que um artigo da linha de Educação e Pesquisa, e que um artigo da 
área de Mercado de Capitais teve 17\% menos citações que um artigo da linha de Educação e Pesquisa.

O gênero do primeiro autor também se mostrou estatisticamente significante para o modelo com um irr $=1,14$. Isso significa que, mantidas constantes as demais condições, um artigo com primeiro autor do sexo feminino recebeu $14 \%$ mais citações que um artigo com primeiro autor do sexo masculino.

A instituição do primeiro autor permaneceu no modelo com três categorias. Mantidas constantes as demais condições, artigos com primeiro autor afiliado à USP tiveram $34 \%$ mais citações e da UFPB tiveram $66 \%$ mais citações que a categoria de referência, que são os artigos com primeiro autor da FECAP.

A Revista em que o artigo foi publicado manteve mais categorias no modelo. A categoria de referência para essa variável era a revista ABCustos. As revistas que mostraram maiores taxas de incidência estimada foram: Revista de Contabilidade \& Finanças (irr = 125,73); Contabilidade Vista \& Revista (irr = 46, 18); e Contabilidade, Gestão e Governança (irr = 6,81). Por outro lado, a Revista Eletrônica de Contabilidade (irr $=0,10$ ), a Revista de Administração e Contabilidade da FAT (irr $=0,16$ ) e a RACEF (irr $=0,18$ ) apresentaram as menores taxas de incidência estimadas.

Os fatores quantitativos retidos no modelo foram o número de referências (irr $=1,01$ ) e a idade da revista no ano em que o artigo foi publicado (irr $=0,82$ ). 0 primeiro achado vai ao encontro do observado na literatura, que afirma que, quanto mais referências um artigo possui, mais citações ele receberá, tendo o irr dessa variável mostrado que essa relação é positiva e quantificada em $1 \%$ por referência extra. A idade da revista mostrou-se um achado contraintuitivo, pois era esperado que houvesse uma relação positiva entre a idade da revista e a quantidade de citações do artigo. No entanto, o irr dessa variável indicou que, mantidas as demais condições constantes, a cada ano a mais de idade que a revista tinha, o número de citações foi $18 \%$ menor.

\subsection{Resultados da Pesquisa Vis-à-vis a Literatura}

O impacto da quantidade de autores sobre as citações foi estatisticamente significante somente na presença da variável idade do artigo e está positivamente relacionado com o aumento do número de citações, na proporção de $5 \%$ mais citações a cada autor adicionado. Esse resultado alinhase com a maior parte da literatura (Leimu \& Koricheva, 2005; Antonakis et al., 2014; Martínez-Blasco et al., 2016; Fox et al., 2016). Em todos os demais modelos, essa variável não se mostrou estatisticamente significante. Dessa forma, considera-se a Hl apenas parcialmente aceita. Pode-se, ainda, cogitar o fato de que as pequenas contribuições, que não são autorias de fato, estão contribuindo para o aumento do número médio de autores, mas não para o resultado final da pesquisa, como alertam Antunes et al. (2014).

O impacto do sexo do primeiro autor sobre as citações mostrou-se estatisticamente significante em seis dos oito modelos elaborados, não sendo estatisticamente significante apenas quando a revista foi removida do modelo e quando a USP foi removida do fator instituição de vínculo do primeiro autor. Os modelos indicam que artigos com primeira autora são de $11 \%$ a $14 \%$ mais citados do que artigos com primeiro autor. Esse resultado mostra-se em concordância 
"Diga-me Quantos te Citam, e Eu te Direi Quem és": Estudo Sobre as Citações no Âmbito da Pesquisa Contábil Brasileira

com a pesquisa de Ayres e Vars (2000). Assim sendo, considera-se a H2 parcialmente aceita.

O impacto da proporção de mulheres autoras na quantidade de citações do artigo não se mostrou estatisticamente significante em nenhum modelo. Dessa forma, considera-se a H3 rejeitada.

O impacto da titulação do primeiro autor na quantidade de citações do artigo mostrou-se relevante quando o primeiro autor era doutor ou mestre, mas apenas na ausência da revista. O modelo indica que artigos com primeiro autor doutor são $27 \%$ mais citados; e artigos com primeiro autor mestre são $21 \%$ mais citados. A categoria de referência são os artigos com primeiro autor bacharel. Esse resultado alinha-se com a pesquisa de Ayres e Vars (2000). Dessa forma, a H4 foi considerada parcialmente aceita.

A afiliação institucional, tratada como instituição do primeiro autor, mostrou-se significante em quase todos os modelos, com variação nas categorias relevantes. Os modelos indicam que, quando a afiliação do primeiro autor era a USP, os artigos eram de $34 \%$ a $44 \%$ mais citados que a categoria de referência. Quando a afiliação do primeiro autor era de uma instituição sem programa de pós-graduação stricto sensu em Contabilidade, os artigos eram de $17 \%$ a $46 \%$ menos citados que a categoria de referência. A categoria de referência era a FECAP.

Na ausência da revista, a afiliação institucional se mostrou mais relevante, dado o seu impacto no número de citações. O impacto da afiliação institucional está alinhado com a literatura (Ayres \& Vars, 2000; Leimu \& Koricheva, 2005; Stremersch et al., 2007; Mingers \& XU, 2010). Diante disso considera-se a H5 aceita.

O impacto da revista na quantidade de citações mostrou-se estatisticamente significante em todos os modelos gerados, embora nem todas as revistas tenham se mostrado estatisticamente significantes. O modelo mais abrangente incluiu 27 das 36 revistas analisadas. O impacto da revista na quantidade de citações está alinhado com a literatura (Leimu \& Koricheva, 2005; Judge et al., 2007; Mingers \& Xu, 2010; Aragão et al., 2014). Dessa forma, considera-se a $\mathrm{H} 6$ aceita.

O impacto do tipo de edição na quantidade de citações não se mostrou estatisticamente significante em nenhum modelo, contradizendo a pesquisa de Martínez-Blasco et al. (2016). Dessa forma, rejeita-se a H7.

O impacto do estrato do Qualis da revista na quantidade de citações de um artigo mostrou-se estatisticamente significante somente na ausência da revista ou idade da revista. Mas, como o Qualis reflete, parcialmente, as taxas médias de citações e tem uma associação não aleatória com as revistas, acaba por se tornar relevante na ausência delas. Diante disso, considera-se a H8 parcialmente aceita.

A idade da revista foi incluída em seis modelos, tendo se mostrado estatisticamente significante em cinco deles, exceto no modelo que incluía a variável idade do artigo. Dessa forma, considera-se a H9 parcialmente aceita.

A influência da posição do artigo dentro da edição mostrou-se estatisticamente insignificante em todos os modelos elaborados. Dessa forma, considera-se a hipótese $\mathrm{H} 10$ rejeitada. Esse fato contraria as pesquisas de Ayres e 
Vars (2000), Judge et al. (2007), Stremersch et al. (2007), que identificaram que ser o primeiro artigo da edição, ou seja, leading article, proporcionava maiores taxas de citação.

O número de termos no título (Ayres \& Vars, 2000), o número de palavraschave (Stremersch et al., 2007; Mingers \& XU, 2010; Stremersch et al., 2015) e o número de páginas (Ayres \& Vars, 2000; Leimu \& Koricheva, 2005; Judge et al., 2007; Stremersch et al., 2007; Mingers \& Xu, 2010; Fox et al., 2016), que a literatura apontava como influentes, não permaneceram em nenhum dos modelos gerados nesta pesquisa. Dessa forma, as hipóteses H11, H12 e H13 foram rejeitadas.

O impacto do número de referências nas citações mostrou-se estatisticamente significante em todos os modelos gerados. Esse achado vai ao encontro da literatura analisada (Judge et al., 2007; Stremersch et al., 2007; Mingers \& XU, 2010; Antonakis et al., 2014; Fox et al., 2016). De fato, os modelos indicam que a cada referência extra, mantidas as demais condições constantes, o artigo tem uma quantidade de citação de 0,2\% a 1,2\% maior. Dessa forma, a $\mathrm{H} 14$ foi aceita.

O impacto da linha de pesquisa nas citações dos artigos mostrou-se estatisticamente significante em todos os modelos gerados, alinhando-se com as pesquisas de Ayres e Vars (2000); Stremersch et al. (2007); Antonakis et al. (2014); Stremersch et al. (2015). Segundo os oito modelos, a linha 1, Contabilidade Gerencial e Controladoria, tem média de citação menor que a categoria de referência, que era a linha 4, Educação e Pesquisa. Segundo cinco modelos, a linha 3, Mercado de Capitais, tem média de citação menor que a categoria de referência. Enquanto os artigos da linha 3 são de 15\% a 19\% menos citados que a linha 4, os artigos da linha 1 são de $26 \%$ e $37 \%$ menos citados que a linha 4 . Dessa forma, a H15 foi aceita.

O impacto do uso de métodos quantitativos nas citações mostrou-se estatisticamente significante apenas na ausência das variáveis idade da revista e estrato Qualis da revista. No entanto, o uso de métodos quantitativos causou redução de $11 \%$ nas citações dos artigos, contrariando a pesquisa de Antonakis et al. (2014), que encontraram uma associação positiva. Esse achado também contraria a média de citações dos dois grupos, já que a média do grupo de artigos com abordagem quantitativa era maior que a do outro grupo. No entanto, há que se considerar que essa variável mostra associação estatisticamente significativa com outras seis variáveis qualitativas, e que são elas que aumentam a média de citações dos artigos dessa categoria. Diante disso, a H16 foi rejeitada.

\section{CONCLUSÃO}

Conforme discutido na revisão de literatura, existe uma perspectiva teórica afirmando que os diversos motivos para que artigos sejam citados transitam entre dois polos: o universalista, que considera fatores, como originalidade dos resultados e rigor metodológico; e o social-construtivista, que abarca fatores, como fama do autor e prestígio do periódico. De acordo com essa perspectiva teórica, colocou-se a questão de pesquisa: Quais fatores do polo social- 
"Diga-me Quantos te Citam, e Eu te Direi Quem és": Estudo Sobre as Citações no Âmbito da Pesquisa Contábil Brasileira

construtivista influenciam as citações que um artigo sobre contabilidade recebe no âmbito da pesquisa contábil brasileira?

Para responder essa questão, fez-se uma coleta de dados de 2.540 artigos, publicados em 36 revistas brasileiras de contabilidade, entre os anos de 2007 e 2012. Para a análise desses dados, utilizou-se uma regressão binomial negativa, e, com base na revisão de literatura, foram elaboradas 16 hipóteses de fatores que influenciam a quantidade de citações dos artigos.

Após a análise, foi possível concluir que o número de citações sofre influência da afiliação institucional do primeiro autor, da revista em que o artigo foi publicado, da linha de pesquisa e do número de referências. A aceitação apenas parcial de algumas hipóteses e a rejeição das demais hipóteses revelam que o cenário da pesquisa contábil brasileira, embora apresente a influência de fatores social-construtivistas, mostra-se bastante flexível quando comparado ao cenário de pesquisa internacional, na área de Negócios, Direito e Sustentabilidade.

Há que se esclarecer que esta pesquisa encontrou limitações e delimitações. Embora tenha-se tentado tratar a pesquisa como populacional, refere-se apenas ao período compreendido pelos triênios 2007-2009 e 2010-2012. Não contempla o quadriênio 2013-2016 nem o seguinte, quando a área de Contabilidade assistiu à implementação de vários programas de pós-graduação, de mestrado e doutorado. Outra limitação consiste no fato de que as revistas brasileiras estão, quase em sua totalidade, fora das grandes bases de dados. Assim, a análise de citações se deu pelo Google Acadêmico. Uma alternativa que poderia ser cogitada como fonte de dados para pesquisas futuras é a base Spell, limitada pelo fato de que várias revistas de Contabilidade ainda não estão incluídas na base.

Como possibilidades de pesquisas futuras, sugere-se o acompanhamento longitudinal do comportamento das citações e dos fatores a elas associados. Outra sugestão é que seja feita uma análise do tipo de citação, como proposto por Stremersch et al. (2015), visando identificar se as citações são realmente usadas para construir a discussão ou se são apenas menções superficiais de trabalhos, que podem nem ter sido lidos. Ou seja, que podem ter sido utilizadas meramente para aumentar o volume de referências bibliográficas. Outras variáveis também podem ser incluídas em estudos futuros, como a legibilidade do paper ou se o artigo teve origem em dissertação ou tese.

\section{REFERÊNCIAS}

Antonakis, J., Bastardoz, N., Liu, Y., \& Schriesheim, C. A. (2014). What makes articles highly cited? The Leadership Quarterly, 25(1), 152-179. doi:10.1016/j.leaqua.2013.10.014

Aragão, I. R. B. N., Oliveira, J. R. S., \& Lima, G. A. S. F. (2014). Ressonância de Artigos e Fator de Impacto de Periódicos Brasileiros de Contabilidade. Revista de Educação e Pesquisa em Contabilidade (REPeC), 8(1), 5-21. doi:10.17524/repec.v8i1.1011 
Ayres, I., \& Vars, F. E. (2000). Determinants of citations to articles in elite law reviews. The Journal of Legal Studies, 29(S1), 427-450. doi:10.1086/468081

Bíblia Sagrada. Evangelho Segundo São Mateus. Recuperado em 14 setembro, 2015 de https://www.bibliaonline.com.br/

Fávero, L. P. L., Belfiori, P, Silva, F. L., \& Chan, B. L. (2009). Análise de dados: modelagem multivariada para tomada de decisões. Rio de Janeiro: Elsevier.

Fávero, L. P. (2015). Análise de dados - Modelos de Regressão com Excel, Stata e Spss. Rio de Janeiro: Elsevier.

Fox, C. W., Paine, C. E., \& Sauterey, B. (2016). Citations increase with manuscript length, author number, and references cited in ecology journals. Ecology and Evolution, 6(21), 7717-7726. doi:10.1002/ece3.2505

Harzing, A. W. (2007) Publish or Perish, available from https://harzing.com/resources/publish-or-perish

Judge, T. A., Cable, D. M., Colbert, A. E., \& Rynes, S. L. (2007). What causes a management article to be cited-article, author, or journal? Academy of Management Journal, 50 (3), 491-506. doi:10.5465/amj.2007.25525577

Law, R., Ye, Q., Chen, W., \& Leung, R. (2009). An analysis of the most influential articles published in tourism journals from 2000 to 2007: A Google Scholar approach. Journal of Travel \& Tourism Marketing, 26(7), 735-746. doi:10.1080/10548400903284628

Leimu, R., \& Koricheva, J. (2005). What determines the citation frequency of ecological papers? Trends in Ecology \& Evolution, 20(1), 28-32. doi:10.1016/j.tree.2004.10.010

Martínez-Blasco, M., Argilés-Bosch, J. M., García-Blandón, J., \& Martínez de lbarreta Zorita, C. (2016). Factores influyentes en las citaciones en contabilidad: un análisis de la REFC. Spanish Journal of Finance and Accounting, 45(4), 487-516. doi: 10.1080/02102412.2016.1216815

Meadows, A. J. (1999). A comunicação científica. Brasília: Briquet de Lemos.

Merton, R. K. (1968). The Matthew effect in science. Science, 159(3810), 56-63. doi: $10.1126 /$ science.159.3810.56

Mingers, J., \& XU, F. (2010). The drivers of citations in management science journals. European Journal of Operational Research, 205(2), 422-430. doi: 10.1016/j.ejor.2009.12.008

Stremersch, S., Verniers, I., \& Verhoef, P. C. (2007). The quest for citations: Drivers of article impact. Journal of Marketing, 71 (3), 171-193. doi:10.1509/jmkg.71.3.171 
"Diga-me Quantos te Citam, e Eu te Direi Quem és": Estudo Sobre as Citações no Âmbito da Pesquisa Contábil Brasileira

Stremersch, S., Camacho, N., Vanneste, S., \& Verniers, I. (2015). Unraveling scientific impact: Citation types in marketing journals. International Journal of Research in Marketing, 32(1), 64-77. doi:10.1016/j.jiresmar.2014.09.004

\section{CONTRIBUIÇÕES DOS AUTORES}

\begin{tabular}{|c|c|c|c|}
\hline Contribuição & $\begin{array}{l}\text { Sandro } \\
\text { Vieira } \\
\text { Soares }\end{array}$ & $\begin{array}{l}\text { Silvia Pereira } \\
\text { de Castro } \\
\text { Casa Nova }\end{array}$ & $\begin{array}{l}\text { Raimundo } \\
\text { Nonato Lima } \\
\text { Filho }\end{array}$ \\
\hline $\begin{array}{l}\text { 1. Idealização e concepção do assunto } \\
\text { e tema da pesquisa }\end{array}$ & $\checkmark$ & $\checkmark$ & $\checkmark$ \\
\hline 2. Definição do problema de pesquisa & $\checkmark$ & $\checkmark$ & $\checkmark$ \\
\hline $\begin{array}{l}\text { 3. Desenvolvimento da Plataforma } \\
\text { Teórica }\end{array}$ & $\checkmark$ & $\checkmark$ & $\checkmark$ \\
\hline $\begin{array}{l}\text { 4. Delineamento da abordagem } \\
\text { metodológica da pesquisa }\end{array}$ & $\checkmark$ & $\checkmark$ & $\checkmark$ \\
\hline 5. Coleta de dados & $\checkmark$ & & \\
\hline $\begin{array}{l}\text { 6. Análises e interpretações dos dados } \\
\text { coletados }\end{array}$ & $\checkmark$ & $\checkmark$ & $\checkmark$ \\
\hline 7. Conclusões da pesquisa & $\checkmark$ & $\checkmark$ & $\checkmark$ \\
\hline 8. Revisão crítica do manuscrito & $\checkmark$ & $\checkmark$ & $\checkmark$ \\
\hline $\begin{array}{l}\text { 9. Redação final do manuscrito, } \\
\text { conforme as normas estabelecidas pela } \\
\text { Revista. }\end{array}$ & $\checkmark$ & $\checkmark$ & $\checkmark$ \\
\hline 10. Orientação & & $\checkmark$ & \\
\hline
\end{tabular}

